\title{
Neuroendocrinology 1980;30:397-398
}

\section{Subject Index}

\section{Accessory sex organs 169 Acetylcholine 329, 344 Adrenal 7, 183}

- cortex 187

medulla 291 Adrenergic blockers 33 Adrenocortical responses 52 Adrenocorticotropin (ACTH) 38, 108, 159.

178, 262, 280, 369 Aging 15 B-Alan;ne thyrotropin-releasing hormone

193 Amizo acids 350 Analgesia 280 Anosmia 169 Anovulation 144 Anterior hypothalamus 33

- pituitary 164

Antidiures;s 275

Anstiestrogens 389

Antihistamines 11

Avoidance 174

-, passive 174

Behavior 174 Bioactivity 355 Blinding 169 Blood-brain barrier 2 Blood pressure 101 Brain monoamines 319

- nuclei 329

Brattleboro rat I 50

Catfish 232

Carbachol 275

Catecholamines 139

Cerebrospinal fluid 206, 382

6-Chloromelatonin 83

Chlorpromazine 319

Choline acetyltransferase 329

Cholinerg ${ }_{i c}$ nerve ending 291

Chronic catheter 323

Orcadian rhythm 291, 369

Cold-water stress 280

Confiuens sinuum 382

Copulatory behavior 220

Copulatory behavior 220
Corticosterone 38, 52, 187, 213, 300, 323,

Corticosterone 38, 52, 187, 213, 300, 323,
369 Corticotropin-releasing factor (CRF) 38,

108, 369 Cyclic adenosine monophosphate (cAMP)

262, 300

- glucose monophosphate (cGMP) 300

Cytidine 285

Delayed puberty 213

Dentate gyrus 187 Dexamethasone 52 Dogs 20, 139 Domperidone 2, 65 Dopam;ne 94, 249, 300, 389 Dopamijergic system 133

Effects of drugs 243 Electrical stimulation 249, 313 p-Endorphin 113, 249, 319 Ergot derivatives 228 Estradiol 15, 144, 329 Estrogen 164, 200,389 Estrus 200 Ethanol 213 Extrahypothalamic areas 122

Fimbrịa 52

Fluoromelatonin 83

Follicle stimulating hormone (FSH) 15,94

164,337

------releasing factor (FSH-RF) 25

Gel chromatography 113 Gonadotropins 25 Growth 243

- hormone (GH) 139, 193, 243, 300, 355,

369,389

- -releasing factor (GH-RF) 319,355

---- secretion 319

\section{Half-life of PLG 377}

Haloperidol 65

Heart rate 101

High-pressure liquid chromatography 377

Hippocampus 52, 187

Histamine 11

Hormone implants 220

6-Hydroxydopamine 362

5-Hydroxytryptophan 101

Hypertonicity 275

Hypertonic stimulation 268

Hypothalamic afferents 238 
norepinephrine 362

- nuclei 113

- pituitary axis 257

Hypothalamus 7, 20, 25, 38, 122, 144, 150,

220, 249, 323 Hypothyroidism 257

mmunoactivity 355

Immunocytochemistry 113, 150,280,285 Infantile rats 25 Intermediate lobe 108 Ions 262 
Kinetics 164 Labeled PLG 377 /i-Lipotropin 280 Lizards 200 Localization 38 Locus ceruleus 300

Luteinizizing hormone (LH) 15, 33, 83, 94, 164, 169, 249, 329, 337, 369

release 362

200, 369

Male rat 94

Maturation 257

Medial basal hypothalamus 94

- preoptic area (MPOA) 144

Medulla 150

Melatonin 83, 169, 382

Metabolism of H-Pro-Leu-Gly-NH

377 Methysergide 133 Mildbrain 337 Milk ejection 313 Mitotic index 88 Modulator 362 Monosodium glutamate 280 Morphine 280 Muscarinic receptors 344

Naloxone 249. 319 Natriuresis 206 Neural control 238 Neuroendocrine 268

- regulation 122

Neurogenịc and systemic stress 323 Neurohypophysịs 268 Neurointermedjate lobe 262 Neuropathology 144 Neurophysịn 150 Neurotransmitters 122 Nitromifene citrate 389 Norepinephrine 33 , 94 , 300 Nucleus lateralis tuberis 232 Nucleus preopticus 232

Optic nerve stimulation 232 Osmoreceptors 206 Ovarian steroids 122 Ovariectomy 15, 45, 164 Ovulation 228, 238 Oxytocin 150, 313

Pain 280

Paired-ion chromatography 377 Paraventriculalar nucleus 150, 285 Pathological hyperprolacținemia 2

398 
Peripheral dopamine receptors 65 Perphenaz;ne 243 Phenothiazines 243 Photoperiod $309 \mathrm{P}_{\mathrm{i}}$ nealectomy 291 Pineal gland 169 Pituitary 7, 25, 313, 389 - gland 70, 76, 344

hormones 70, 76

- lobes 113

- melanocyte-stimulating hormone (MSH) 309

- stalk section 70, 76

- tumors 389 Plasma 33

- luteinizing hormone 45 Posterior pituitary 108 Preoptic area 94 Progesterone 15, 183, 329

Prolactin 2, 7, 65, 83, 133, 139, 159, 169, 213, 249, 300, 337, 350, 369, 389

- release 11

Prolyl-leucyl-glycinamide 377 Pseudopregnancy 159 Puberty 20,213

Puerperal hyperprolactinemia 2 Pulsatile LH release 122 
Quinuclidinyḷ benzịlate 344

Rabbit 362 Radioautography 285 Radiofrequency lesions 88 Radioimmunoassay 113,382 Rat 7, 228, 313 Reflex ovulation 362 Releasing hormone 344 Renin 101 Reptiles 200 Rhesus monkey 249

Seasonal variation 309 Serotonin 101, 139, 228

- antagonists 228

Serotoninergic system 133

Serum anti-ariginine vasopressin 178 Sexual behavior 20

- receptivity 200

Sheep 275

Somatostatin 319, 369 Spinal cord 150 Stress 159, 187, 300

- response 38

Synaptic vesicle number 291 
Tamoxifen 389 Taurine 350 Testes 183

Testicular weights 169 Testosterone 7, 20, 94, 183, 329 Thalamic pigeon 323 Thyrotropin-releasing hormone (TRH)

139, 193, 200, 369 Thyroid-stimulating hormone (TSH) 193 Transection 337 Tryptophan 101 Tricyclic compounds 228 Tumor 7

Ultradian rhythm 291 Unit activity 268 Uterine contractions 313

Vasopressin 108, 150, 174, 178, 268, 285 - antidiuretic hormone 206 Ventral medial tegmental area 300

Yoshiada ascites tumor 88 